\title{
Some Properties on Fréchet-Weibull Distribution with Application to Real Life Data
}

\author{
Deepshikha Deka $^{1, *}$, Bhanita Das ${ }^{1}$, Bhupen K Baruah ${ }^{2}$, Bhupen Baruah ${ }^{2}$ \\ ${ }^{1}$ Department of Statistics, North-Eastern Hill University, Shillong-793022, Meghalaya, India \\ ${ }^{2}$ Department of Chemistry, Jagannath Barooah College, Jorhat-785001, Assam, India
}

Received October 20, 2020; Revised December 16, 2020; Accepted January 20, 2021

\begin{abstract}
Cite This Paper in the following Citation Styles
(a): [1] Deepshikha Deka, Bhanita Das, Bhupen K Baruah, Bhupen Baruah, "Some Properties on Fréchet-Weibull Distribution with Application to Real Life Data," Mathematics and Statistics, Vol. 9, No. 1, pp. 8 - 15, 2021. DOI: 10.13189/ms.2021.090102.
\end{abstract}

(b): Deepshikha Deka, Bhanita Das, Bhupen K Baruah, Bhupen Baruah (2021). Some Properties on Fréchet-Weibull Distribution with Application to Real Life Data. Mathematics and Statistics, 9(1), 8 - 15. DOI: 10.13189/ms.2021.090102.

Copyright $\subseteq 2021$ by authors, all rights reserved. Authors agree that this article remains permanently open access under the terms of the Creative Commons Attribution License 4.0 International License

\begin{abstract}
Research, development and extensive use of generalized form of distributions in order to analyze and modeling of applied sciences research data has been growing tremendously. Weibull and Fréchet distribution are widely discussed for reliability and survival analysis using experimental data from physical, chemical, environmental and engineering sciences. Both the distributions are applicable to extreme value theory as well as small and large data sets. Recently researchers develop several probability distributions to model experimental data as these parent models are not adequate to fit in some experiments. Modified forms of the Weibull distribution and Fréchet distribution are more flexible distributions for modeling experimental data. This article aims to introduce a generalize form of Weibull distribution known as Fréchet-Weibull Distribution (FWD) by using the T-X family which extends a more flexible distribution for modeling experimental data. Here the $p d f$ and $c d f$ with survival function $[\mathrm{S}(\mathrm{t})]$, hazard rate function $[\mathrm{h}(\mathrm{t})]$ and asymptotic behaviour of $p d f$ and survival function and the possible shapes of $p d f, c d f, \mathrm{~S}(\mathrm{t})$ and $\mathrm{h}(\mathrm{t})$ of FWD have been studied and the parameters are estimated using maximum livelihood method (MLM). Some statistical properties of FWD such as mode, moments, skewness, kurtosis, variation, quantile function, moment generating function, characteristic function and entropies are investigated. Finally the FWD has been applied to two sets of observations from mechanical engineering and shows the superiority of FWD over other related distributions. This study will provide a useful tool to analyze and modeling of
\end{abstract}

datasets in Mechanical Engineering sciences and other related field.

Keywords Weibull Distribution, Fréchet Distribution, T-X Family, Properties, Applications

\section{Introduction}

Observations on physical sciences, environmental sciences, medical sciences, engineering sciences etc. generate large data sets. Estimation of observable parameters, their analysis, interpretation and modelling of these parameters are now getting more importance. Weibull distribution is an important statistical distribution widely used in reliability and survival analysis. It has several applications using extreme value theory (Gumbel [9]). Studies reveals that Weibull distribution is good fit to experimental data of engineering and physics (Kapoor and Lamberson [10]), quality control (Nelson and Wayne [15]) and medicine (Sica and Luko [19]). Lai et al. [11] have introduced Weibull distributions and their applications. Therefore, researchers in the last few years developed various extensions and modified forms of the Weibull distribution to obtain more flexible distributions for modeling experimental data. For example Ahmad et al. [1] have discussed generalized form of new Extended Weibull distribution, Pobocikova et al. [17] have introduced transmuted Weibull distribution along with its 
applications. Cordeiro et al. [6], [7], has introduced some other form of generalization of Weibull distribution such as Lindly Weibull distribution and Transmuted Generalized Modified Weibull distribution and Gumbel Weibull distribution (Al-Aqtash et al. [2]).

Fréchet distribution is an important distribution applied in extreme value theory for earthquake and flood data analysis. Silva et al. [18] has introduced the gamma extended Fréchet distribution, Nadarajah and Kotz [14] introduced the exponentiated Fréchet distribution, Mead et al. [13] introduced the beta exponential Fréchet distribution. The Transmuted Fréchet distribution was developed by Mahmoud and Mandouh [12]. Properties and applications of six parameters Fréchet distribution was studied by Yousof et al. [23].

Research and development on generalized form of distributions and their extensive use in order to modelling of applied sciences research data has been growing tremendously. Researchers develop several probability distributions to model such kind of experimental data as the parent model is not adequate to fit experimental data. Review of literature reveals that Weibull and Fréchet distributions have been used as a sophisticated statistical distribution and found application in several real life situations for both small and large data sets. The main objective of this study is to develop a generalized form of Weibull distribution called Fréchet-Weibull distribution (FWD) using new T-X family method (Alzaatreh et al. [3]).

The probability density function ( $p d f)$ and the cumulative distribution function $(c d f)$ of Weibull distribution are respectively given by

$$
\begin{gathered}
d(x)=\frac{k}{\lambda}\left(\frac{x}{\lambda}\right)^{k-1} \exp \left(-\left(\frac{x}{\lambda}\right)^{k}\right) ; \\
x>0, \lambda>0, k>0 \\
\text { and } D(x)=1-\exp \left(-\left(\frac{x}{\lambda}\right)^{k}\right) ; \\
x>0, \lambda>0, k>0
\end{gathered}
$$

The probability density function (pdf) and the cumulative distribution function $(c d f)$ of Fréchet distribution are given by

$$
\begin{gathered}
m(x)=\frac{\alpha}{\beta}\left(\frac{\beta}{x}\right)^{\alpha+1} \exp \left(-\left(\frac{\beta}{x}\right)^{\alpha}\right), \\
x>0, \beta>0, \alpha>0 \\
\text { and } M(x)=\exp \left(\left(-\frac{\beta}{x}\right)^{\alpha}\right), \\
x>0, \beta>0, \alpha>0
\end{gathered}
$$

respectively.

According to Alzaatreh et al. [3], if $X$ be a random variable with $p d f f(x)$ and $c d f F(x)$ and $T$ be a continuous random variable with $p d f r(t)$ defined on $[a, b]$, then the $c d f$ of a new family of distributions is defined as

$$
G(x)=\int_{a}^{W(F(x))} r(t) d t
$$

Where $W(F(x))$ satisfies the following conditions:

(i) $W(F(x)) \in[a, b],-\infty \leq a<b \leq \infty$

(ii) $W(F(x))$ is differentiable and monotonically non-decreasing.

(iii) $W(F(x)) \rightarrow \mathrm{a}$ as $x \rightarrow-\infty$ and $W(F(x)) \rightarrow \mathrm{b}$ as $x \rightarrow \infty$

Also $W(x)=-\log (1-F(x))$.

The $c d f$ of $G(x)$ in (1) can be written as $G(x)=$ $R[W(F(x))]$, where $R(t)$ is $c d f$ of the random variable $T$. The corresponding $p d f$ associated with (1) is written as,

$$
g(x)=\left\{\frac{d}{d x} W(F(x))\right\} r(W(F(x)))
$$

Considering the random variable $X$ follows Weibull distribution, thus for Weibull distribution $W[F(x)]=$ $-\log (1-D(x))=\left(\frac{x}{\lambda}\right)^{k}$ and the random variable $T$ follows Fréchet distribution. As a result we can obtain the Fréchet-Weibull distribution (FWD).

The parameters of the FWD are estimated by using maximum likelihood method. The $p d f$ and $c d f$ along with survival function, hazard rate function, reverse hazard rate function, asymptotic behavior of $p d f$ and $\mathrm{S}(\mathrm{t})$, and the possible shapes of $p d f, c d f, \mathrm{~S}(\mathrm{t})$ and $\mathrm{h}(\mathrm{t})$ of FWD have been studied. Statistical properties such as moments, skewness, kurtosis, variation, quantile function, mode, moment generating function, characteristic function are also studied. The entropies of FWD such as Renyi, Tsallis and Shannon entropies and order statistics of FWD are introduced. Finally we have applied the FWD to two real life data sets viz., breaking stress of carbon fibers (Nichols and Padgett, [16]) and strength of glass fibers Smith and Naylor [21].

\section{The Fréchet-Weibull Distribution (FWD)}

The $c d f$ of a random variable $X$ which follows FWD with parameters $\alpha, \beta, \lambda, k$ is obtained as

$$
\begin{gathered}
F(x)=\exp \left(-\beta^{\alpha}\left(\frac{\lambda}{x}\right)^{\alpha k}\right), x>0, \alpha>0, \\
\beta>0, \lambda>0, k>0
\end{gathered}
$$

and its corresponding $p d f$ is obtained as

$$
\begin{gathered}
f(x)=\alpha k \beta^{\alpha} \lambda^{\alpha k} x^{-1-\alpha k} \exp \left(-\beta^{\alpha}\left(\frac{\lambda}{x}\right)^{\alpha k}\right), \\
x>0, \alpha>0, \beta>0, \lambda>0, k>0
\end{gathered}
$$

where $\alpha$ and $k$ are shape parameters and $\lambda$ and $\beta$ are scale parameters. 


\section{Survival [S(t)], Hazard Rate [h(t)] and Reverse Hazard Rate Function [r(t)] of FWD}

The survival function $S(t)$, which is the probability of an item not failing prior to some time $t$ is defined by $S(t)=1-F(t)$. Thus using equation (3) we derive the survival function $S(t)$ of FWD as

$$
\begin{gathered}
S(t)=1-F(t)=1-\exp \left(-\beta^{\alpha}\left(\frac{\lambda}{t}\right)^{\alpha k}\right) ; \\
t>0, \alpha>0, \beta>0, k>0
\end{gathered}
$$

The other characteristic of interest of a random variable is the hazard rate function defined by $h(t)=\frac{f(t)}{s(t)}$, which is an important quality characterizing life phenomenon. It can be loosely interpreted as the conditional probability of failure, given it has been survived to time $t$.Thus using equations (4) and (5) we derive the hazard rate function $h(t)$ of FWD as

$$
\begin{gathered}
h(t)=\frac{f(t)}{S(t)}=\frac{\alpha k \beta^{\alpha} \lambda^{\alpha k} t^{-1-\alpha k} \exp \left(-\beta^{\alpha}\left(\frac{\lambda}{t}\right)^{\alpha k}\right)}{1-\exp \left(-\beta^{\alpha}\left(\frac{\lambda}{t}\right)^{\alpha k}\right)} ; \\
t>0, \alpha>0, \beta>0, k>0
\end{gathered}
$$

The reverse hazard rate function is the ratio of the probability density to its distribution function and is defined by $r(t)=\frac{f(t)}{F(t)}$, and has applications in the field of engineering, actuarial science, forensic science and similar other field (Block et al. [5], Sengupta et al. [18]). Thus by using equations (3) and (4) we derive the reverse hazard rate function of FWD as

$$
\begin{gathered}
r(t)=\frac{f(t)}{F(t)}=\alpha k \beta^{\alpha} \lambda^{\alpha k} t^{-1-\alpha k} ; t>0, \\
\alpha>0, \beta>0, k>0
\end{gathered}
$$

\section{Asymptotic Behavior}

The limit of $p d f$ for FWD at $x=0$ and $x=\infty$ are given by

$$
\lim _{x \rightarrow 0} f(x)=\alpha k \beta^{\alpha} \lambda^{\alpha k} \lim _{x \rightarrow 0} x^{-1-\alpha k} \exp \left(-\beta^{\alpha}\left(\frac{\lambda}{x}\right)^{\alpha k}\right)=0
$$

respectively, while the limits of survival function of the same points are given respectively by

$$
\lim _{t \rightarrow 0} S(t)=1 \text { and } \lim _{t \rightarrow \infty} S(t)=0 .
$$

\section{The Graphical Representation of $p d f, c d f, S(t)$ and} $h(t)$ of FWD

For some known values of the parameters $\alpha, \beta, \lambda$ and $k$; the possible shapes of the $p d f, c d f, S(t)$ and $h(t)$ of FWD are presented in the Figure 1, Figure 2, Figure 3 and Figure 4 respectively.

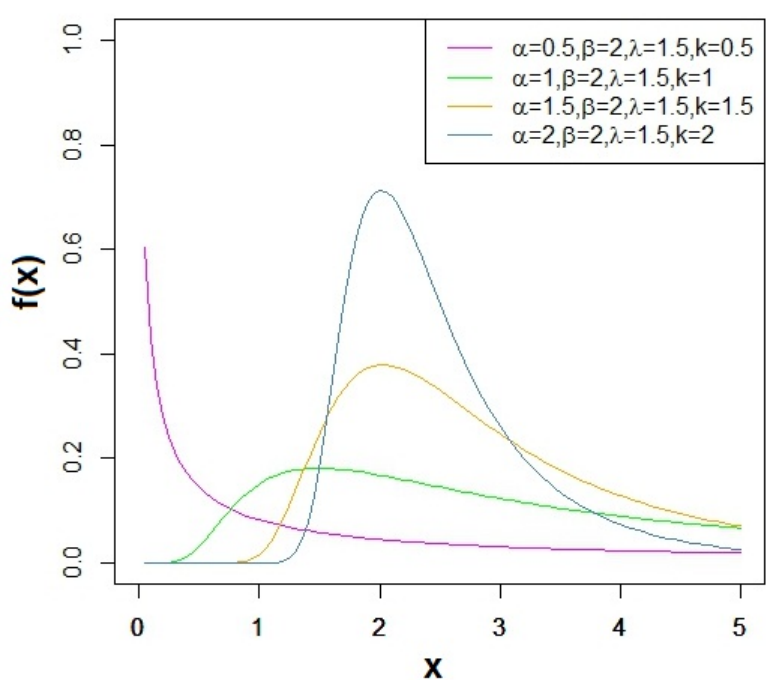

Figure 1. $p d f$ of FWD for selected values of the parameters

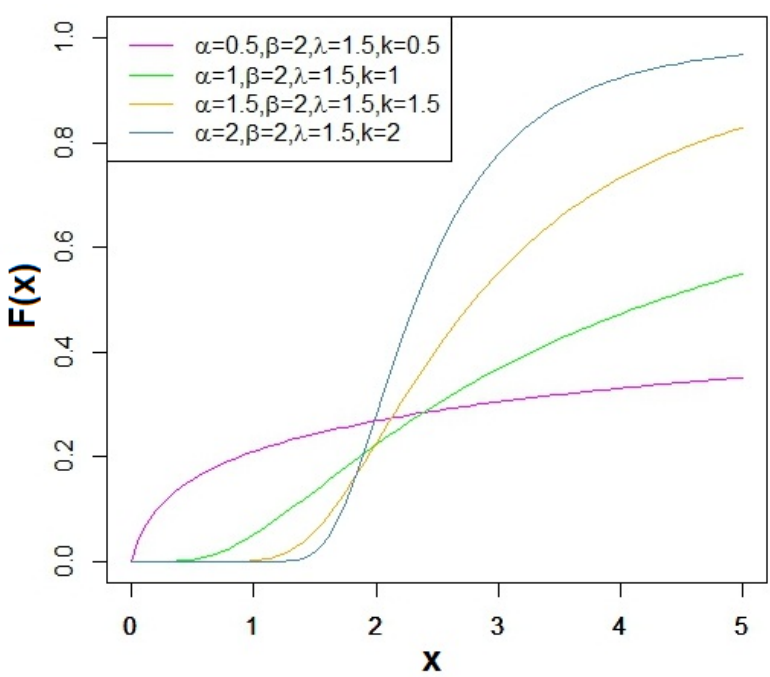

Figure 2. $c d f$ of FWD for some selected values of the parameters

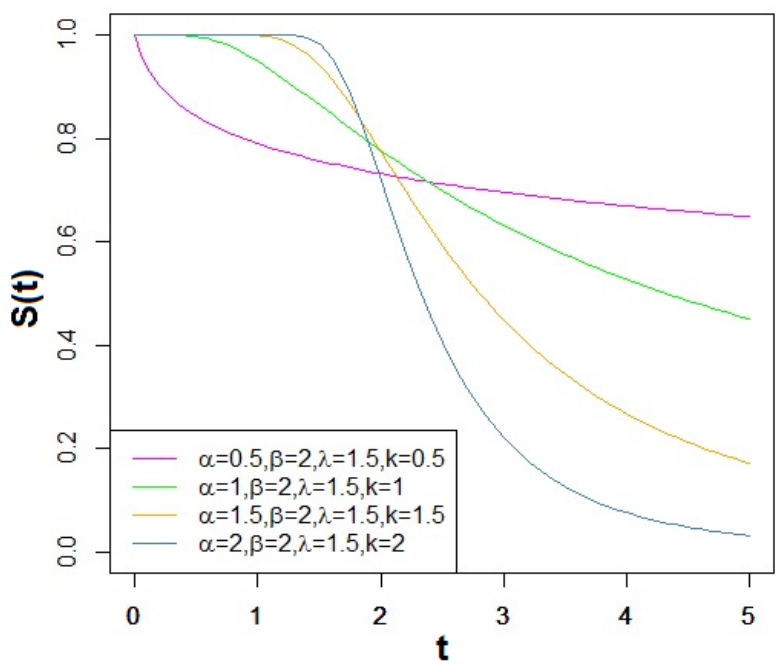

Figure 3. Survival function, $S(t)$, of FWD 


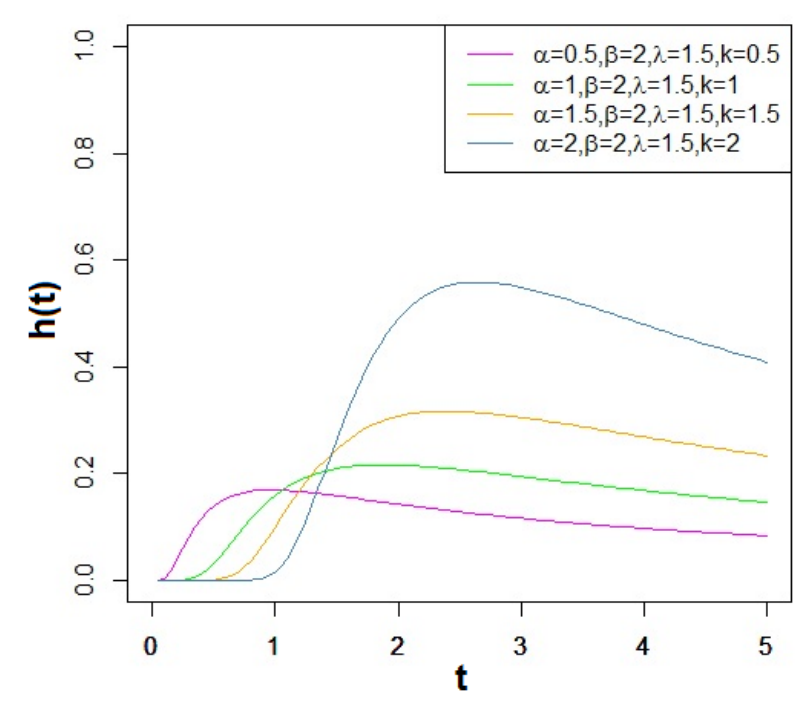

Figure 4. Hazard rate function, $h(t)$, of FWD

\section{Statistical Properties}

\section{Moments of FWD}

The $r^{\text {th }}$ moments $\mu_{r}^{\prime}$ about the origin of FWD is given by

$$
\begin{gathered}
\mu_{r}^{\prime}=E\left(X^{r}\right)=\int_{0}^{\infty} x^{r} f(x) d x=\lambda^{r} \beta^{\frac{r}{k}} \Gamma\left(1-\frac{r}{\alpha k}\right), \\
\mathrm{r}<\alpha k
\end{gathered}
$$

by setting $r=1,2,3$ and 4 in the above equation we obtain the first four moments about the origin of FWD respectively. The mean and variance of FWD are:

$$
\mu_{1}^{\prime}=\mu=\lambda \beta^{\frac{1}{k}} \Gamma\left(1-\frac{1}{\alpha k}\right)
$$

Also

$$
\mu_{2}^{\prime}=\lambda^{2} \beta^{2 / k} \Gamma\left(1-\frac{2}{\alpha k}\right)
$$

$\therefore$ Variance $\sigma^{2}=\mu_{2}^{\prime}-\mu_{1}^{/ 2}=\lambda^{2} \beta^{2 / k}\left[\Gamma\left(1-\frac{2}{\alpha k}\right)-\right.$ $\left.\left(\Gamma\left(1-\frac{1}{\alpha k}\right)\right)^{2}\right]$

respectively, and the first four central moments about the mean for FWD can be obtained by the following relations:

$$
\begin{gathered}
\mu_{1}=\mu_{1}^{\prime}-\mu=0 \\
\mu_{2}=\mu_{2}^{\prime}-\mu_{1}^{/ 2}=\lambda^{2} \beta^{2 / k}[\Gamma(1-2 / \alpha k) \\
\left.-(\Gamma(1-1 / \alpha k))^{2}\right]
\end{gathered}
$$

$$
\begin{aligned}
\mu_{3} & =\mu_{3}^{\prime}-3 \mu_{2}^{\prime} \mu_{1}^{\prime}+2 \mu_{1}^{/ 2} \\
& =\lambda^{3} \beta^{3 / k}[\Gamma(1-3 / \alpha k) \\
& -3 \Gamma(1-2 / \alpha k) \Gamma(1-1 / \alpha k) \\
& \left.+2(\Gamma(1-1 / \alpha k))^{3}\right] \\
\mu_{4}= & \mu_{4}^{\prime}-4 \mu_{3}^{\prime} \mu_{1}^{\prime}+6 \mu_{2}^{\prime} \mu_{1}^{/ 2}-3 \mu_{1}^{/ 4} \\
= & \lambda^{4} \beta^{4 / k}[\Gamma(1-4 / \alpha k) \\
& +\Gamma(1-1 / \alpha k)[-4 \Gamma(1-3 / \alpha k) \\
& +6 \Gamma(1-2 / \alpha k) \Gamma(1-1 / \alpha k) \\
& \left.\left.-3(\Gamma(1-1 / \alpha k))^{3}\right]\right]
\end{aligned}
$$

respectively. Using the above expressions we obtain the expression for coefficient of skewness, coefficient of kurtosis and coefficient of variation of FWD respectively as follows:

$$
\begin{gathered}
\beta_{1}=\frac{\mu_{3}^{2}}{\mu_{2}^{3}} \\
=\frac{\left(\Gamma(1-3 / \alpha k)-3 \Gamma(1-2 / \alpha k) \Gamma(1-1 / \alpha k)+2(\Gamma(1-1 / \alpha k))^{3}\right)^{2}}{\left(\Gamma(1-2 / \alpha k)-(\Gamma(1-1 / \alpha k))^{2}\right)^{3}} \\
\beta_{2}=\frac{\mu_{4}}{\mu_{2}^{2}} \\
=-3 \\
+\frac{\Gamma(1-4 / \alpha k)+3(\Gamma(1-2 / \alpha k))^{2}-4 \Gamma(1-3 / \alpha k) \Gamma(1-1 / \alpha k)}{\left(\Gamma(1-2 / \alpha k)-(\Gamma(1-1 / \alpha k))^{2}\right)^{2}} \\
C V=\frac{\sigma}{\mu} \times 100=\frac{\sqrt{\Gamma(1-2 / \alpha k)-(\Gamma(1-1 / \alpha k))^{2}}}{\Gamma(1-1 / \alpha k)} \times 100
\end{gathered}
$$

\section{Mode of FWD:}

By taking the logarithm of $p d f(4)$ and differentiating it with equality to zero, we have the mode of FWD as following

$$
x_{0}=\lambda\left(\frac{\alpha k \beta^{\alpha}}{1+\alpha k}\right)^{\frac{1}{\alpha k}}
$$

\section{Quantile Function of FWD:}

The quantile function ( $q f) Q(p)$ of FWD is given by

$$
Q(p)=\inf \{x \in R: F(x) \geq p\}=\lambda\left(-\beta^{\alpha} \ln p\right)^{-\frac{1}{\alpha k}}
$$

by setting $p=0.25,0.5$ and 0.75 in equation (8) we obtain first, second and third quantiles of FWD respectively. 


\section{Moment Generating Function and Characteristics Function of FWD:}

The moment generating function of FWD is obtained as

$$
M(t)=\sum_{m=0}^{\infty} \frac{t^{m}}{m !} \lambda^{m} \beta^{m / k} \Gamma(1-m / \alpha k)
$$

and the characteristic function of FWD is obtained as

$$
\phi(t)=\sum_{m=0}^{\infty} \frac{(i t)^{m}}{m !} \lambda^{m} \beta^{m / k} \Gamma(1-m / \alpha k)
$$

\section{Entropy}

The entropy of a random variable is a measure of uncertainty variation and has been used in different situations in science and engineering. Here we have derived Renyi $R_{r}(X)$, Tsallis $T_{r}(X)$ and Shannon $S_{H}(X)$ entropies (Tabass [22])of FWD and are obtained as

$$
\begin{aligned}
& =\frac{1}{1-r} \log \int_{0}^{\infty} f^{r}(x) d x \\
& =\frac{1}{1-r} \log \left[\left(\frac{\alpha k}{\lambda}\right)^{r-1} \beta^{\frac{1}{k}(1-r)} r^{\frac{1}{\alpha k}-\frac{r}{\alpha k}-r} \Gamma\left(\frac{r}{\alpha k}+r-\frac{1}{\alpha k}\right)\right], \\
& r>0, r \neq 1
\end{aligned}
$$

$$
\begin{gathered}
T_{r}(X)=-R_{r}(X)-1 \\
S_{H}(X)=-\int_{0}^{\infty} f(x) \log f(x) d x \\
=\lim _{r \rightarrow 1} R_{r}(X) \\
=1+\log \lambda+\frac{1}{k} \log \beta-\log \alpha k \\
+\left(1+\frac{1}{\alpha k}\right)(\gamma)
\end{gathered}
$$

respectively, where $\gamma=-\int_{0}^{\infty} e^{-x} \ln (x) d x$, which is Euler Mascheroni constant.

\section{Order Statistics}

\section{$p d f$ and $c d f$ of $\boldsymbol{i}^{\text {th }}$ Order Statistics of FWD:}

Let $X_{1}, X_{2}, \ldots, X_{n}$ is a random sample of size $n$ from FWD. Let $X_{1: n}<X_{2: n}<\cdots<X_{n: n}$ denote the corresponding order statistics. The $p d f$ and the $c d f$ of the $i^{\text {th }}$ order statistics of FWD are given by

$$
\begin{aligned}
f_{i: n}(x) & =\lim _{\delta x \rightarrow 0} \frac{P\left(x<X_{i: n} \leq x+\delta x\right)}{\delta x} \\
& =\frac{n !}{(i-1) !(n-i) !}(F(x))^{i-1}[1 \\
& -F(x)]^{n-i} f(x)
\end{aligned}
$$

$$
\begin{gathered}
=\frac{n ! \alpha k \beta^{\alpha} \lambda^{\alpha k}}{(i-1) !(n-i) !} x^{-1-\alpha k} \exp \left(-i \beta^{\alpha}\left(\frac{\lambda}{x}\right)^{\alpha k}\right)(1 \\
\left.-\exp \left(-\beta^{\alpha}\left(\frac{\lambda}{x}\right)^{\alpha k}\right)\right)^{n-i}
\end{gathered}
$$

and

$$
\begin{aligned}
F_{i: n}(x)= & \sum_{i=1}^{n}\left(\begin{array}{l}
n \\
r
\end{array}\right)(F(x))^{r}(1-F(x))^{n-r} \\
& =\sum_{r=1}^{n}\left(\begin{array}{l}
n \\
r
\end{array}\right) \exp \left(-r \beta^{\alpha}\left(\frac{\lambda}{x}\right)^{\alpha k}\right)(1 \\
& \left.-\exp \left(-\beta^{\alpha} \frac{\lambda^{\alpha k}}{x}\right)\right)^{n-r}
\end{aligned}
$$

respectively, by setting $i=1$ we can obtain the distribution of the minimum order statistics and by setting $i=n$ we can obtain the distribution of the maximum order statistics of FWD.

\section{Limiting Distribution for Maximum Order Statistics}

Suppose that $Z_{n}=X_{n: n}=\max \left(X_{1}, X_{2}, \ldots, X_{n}\right)$ from FWD and the limiting distribution of $Z_{n}$ can be obtained by the theorem (2.1.1) in Galambos [8] as following:

$$
\lim _{t \rightarrow \infty} \frac{1-F(t x)}{1-F(t)}=x^{-\alpha k}
$$

The $\lim _{n \rightarrow \infty} P\left(Z_{n}<a_{n}+b_{n} x\right)=\exp \left(-x^{-\alpha k}\right), x>0$ and the normalizing constants are

$$
\begin{aligned}
a_{n} & =0 \text { and } b_{n}=F^{-1}\left(1-\frac{1}{n}\right) \\
& =\lambda\left(-\beta^{-\alpha} \ln \left(1-\frac{1}{n}\right)\right)^{-\frac{1}{\alpha k}}
\end{aligned}
$$

\section{Estimation of Parameters}

There are several methods for the estimation of parameters of a newly develop distribution. Among these methods, the maximum likelihood method is most commonly used. So, we consider the estimation of the parameters $\alpha, \beta, \lambda$ and $k$ of the FWD from complete sample by the method of maximum likelihood. Let $X_{1}, X_{2}, \ldots, X_{n}$ be a random sample of size $n$ drawn from $X$ having $p d f$ (4), then the log-likelihood function is given by

$$
\begin{gathered}
\log L(x)=n \log \alpha+n \log k+n k \log \beta+n \alpha k \log \lambda- \\
\beta^{\alpha} \lambda^{\alpha k} \sum_{i=1}^{n} x_{i}^{-\alpha k}-(1+\alpha k) \sum_{i=1}^{n} \log x_{i}
\end{gathered}
$$

The first derivative of equation (9) w.r.t. the parameters $\alpha, \beta, \lambda$ and $k$ are expressed in equation (10),(11),(12) and 
(13) respectively.

$$
\begin{gathered}
\frac{\partial \log L(x)}{\partial \alpha}=\frac{n}{\alpha}+n \log \beta+n k \log \lambda-k \sum_{i=1}^{n} \log x_{i}- \\
\beta^{\alpha} \log \beta \sum_{i=1}^{n}\left(\frac{\lambda}{x_{i}}\right)^{\alpha k}-k \beta^{\alpha} \sum_{i=1}^{n}\left(\frac{\lambda}{x_{i}}\right)^{\alpha k} \log \left(\frac{\lambda}{x_{i}}\right) \\
\frac{\partial \log L(x)}{\partial \beta}=\frac{n \alpha}{\beta}-\alpha \beta^{\alpha-1} \lambda^{\alpha k} \sum_{i=1}^{n} x_{i}^{-\alpha k} \\
\frac{\partial \log L(x)}{\partial \lambda}=\frac{n \alpha k}{\lambda}-\alpha k \beta^{\alpha} \lambda^{\alpha k-1} \sum_{i=1}^{n} x_{i}^{-\alpha k} \\
\frac{n}{k}+n \alpha \log \lambda-\alpha \beta^{\alpha} \sum_{i=1}^{n}\left(\frac{\lambda}{x_{i}}\right)^{\alpha k} \log \left(\frac{\lambda}{x_{i}}\right)-\alpha \sum_{i=1}^{n} \log x_{i}
\end{gathered}
$$

Setting equation (10), (11), (12) and (13) to zero and solving them simultaneously yields the maximum likelihood estimates of the parameters of FWD.

\section{Simulation Study}

Let $\theta=(\alpha, \beta, \lambda, k)^{/}$denote the vector of parameters of FWD. Random samples of size $n=$ $50,100,200$ and 400have been generated from FWD with known values of $\theta$. We take $\alpha=1.8, \beta=1.5, \lambda=$ 2 and $k=2.5$.The generated data sets have been fitted to FWD by the method of maximum likelihood. Table 1 shows the estimates and mean square errors (MSEs) of the parameters of FWD for each sample size.

Table 1. Estimates and Mean Square Errors (MSEs) of $\hat{\alpha}, \hat{\beta}, \hat{\lambda}$ and $\hat{k}$

\begin{tabular}{|c|c|c|}
\hline$n$ & Estimates & MSE \\
\hline \multirow{4}{*}{50} & $\hat{\alpha}=1.951478$ & 0.9377901 \\
\cline { 2 - 3 } & $\hat{\beta}=1.938345$ & 1.1611402 \\
\cline { 2 - 3 } & $\hat{\lambda}=2.496579$ & 0.4318672 \\
\cline { 2 - 3 } & $\hat{k}=2.651416$ & 0.5948734 \\
\hline \multirow{4}{*}{100} & $\hat{\alpha}=1.900678$ & 0.9107752 \\
\cline { 2 - 3 } & $\hat{\beta}=1.709278$ & 0.9568072 \\
\cline { 2 - 3 } & $\hat{\lambda}=2.257952$ & 0.3792981 \\
\cline { 2 - 3 } & $\hat{k}=2.603404$ & 0.4733069 \\
\hline \multirow{4}{*}{200} & $\hat{\alpha}=1.729510$ & 0.7119531 \\
\cline { 2 - 3 } & $\hat{\beta}=1.514060$ & 0.6981372 \\
\cline { 2 - 3 } & $\hat{\lambda}=1.871951$ & 0.2263387 \\
\cline { 2 - 3 } & $\hat{k}=2.536961$ & 0.3506112 \\
\hline \multirow{4}{*}{400} & $\hat{\alpha}=1.696739$ & 0.5419531 \\
\cline { 2 - 3 } & $\hat{\beta}=1.364894$ & 0.4571365 \\
\cline { 2 - 3 } & $\hat{\lambda}=1.668777$ & 0.1273043 \\
\cline { 2 - 3 } & $\hat{k}=2.443872$ & 0.2010734 \\
\hline
\end{tabular}

From the figures of Table 1, we have seen that estimates are close to their actual values and as the sample size increases, the MSE decreases.

\section{Real Life Applications}

To study the goodness of fit of the newly developed FWD, we have considered two different data sets related to mechanical properties of carbon fiber (Nichols and Padgett, [16]) and glass fibers (Smith and Naylor [21]). The secondary data sets are given below:

Dataset 1: It refers to 100 observations of breaking stress of carbon fibers

$3.70,2.74,2.73,2.50,3.60,3.11,3.27,2.87,1.47,3.11$, $4.42,2.41,3.19,3.22,1.69,3.28,3.09,1.87,3.15,4.90$, $3.75,2.43,2.95,2.97,3.39,2.96,2.53,2.67,2.93,3.22$, 3.39, 2.81, 4.20, 3.33, 2.55, 3.31, 3.42, 2.85, 2.56, 3.56, $3.15,2.35,2.55,2.59,2.38,2.81,2.77,2.17,2.83,1.92$, $1.41,3.68,2.97,1.36,0.98,2.76,4.91,3.68,1.84,1.59$, $3.19,1.57,0.81,5.56,1.73,1.59,2.00,1.22,1.12,1.71$, $2.17,1.17,5.08,2.48,1.18,3.51,2.17,1.69,1.25,4.38$, $1.84,0.39,3.68,2.48,0.85,1.61,2.79,4.70,2.03,1.80$, $1.57,1.08,2.03,1.61,2.12,1.89,2.88,2.82,2.05,3.65$.

Dataset2: It refers to 63 observations of the strength of 1.5 cm diameter glass fibers:

$0.55,0.93,1.25,1.36,1.49,1.52,1.58,1.61,1.64,1.68$, $1.73,1.81,2.04,0.74,1.04,1.27,1.39,1.49,1.53,1.59$, $1.61,1.66,1.68,1.76,1.82,2.01,0.77,1.11,1.28,1.42$, $1.51,1.54,1.6,1.62,1.66,1.69,1.76,1.84,2.24,0.81$, $1.31,1.29,1.48,1.5,1.55,1.61,1.62,1.66,1.7,1.77,1.84$, $0.84,1.24,1.3,1.48,1.51,1.55,1.61,1.63,1.67,1.7,1.78$, 1.89 .

Using fitdistrplus package in $\mathrm{R}$ we have studied the goodness of fit of the data to the newly developed FWD along with generalized new extended weibull distribution (GNEXWD) (Ahmad et al. [1]), Lindley Weibull distribution (Cordeiro et al. [6]) and the half-logistic generalized Weibull distribution (HLGWD) (Anwar and Bibi[4]).

Tables 2 and Table 3 represents the maximum likelihood estimates of the parameters together with the log-likelihood ( $-2 l$ ), Akaike Information Criterion (AIC), Bayesian Information Criterion (BIC) and Corrected Information Criterion (CAIC) values for the data sets, respectively.

From Table 2 and Table 3, it is seen that the proposed Fréchet-Weibull distribution (FWD) provides better fit to the datasets under consideration in terms of all criteria, namely the log-likelihood, the AIC as well as the BIC and the CAIC. 
Table 2. Estimated-2l, AIC, BIC, and CAIC for Dataset 1

\begin{tabular}{|c|c|c|c|c|c|}
\hline Distribution & Estimates & $-2 l$ & AIC & BIC & CAIC \\
\hline \multirow{4}{*}{ FWD } & $\hat{\alpha}=0.76549$ & \multirow{4}{*}{301.973} & \multirow{4}{*}{309.973} & \multirow{4}{*}{320.393} & \multirow{4}{*}{310.394} \\
\hline & $\hat{\beta}=2.62019$ & & & & \\
\hline & $\hat{\lambda}=1.90099$ & & & & \\
\hline & $\hat{k}=0.96361$ & & & & \\
\hline \multirow{4}{*}{ GNEXWD } & $\hat{\alpha}=2.8138$ & \multirow{4}{*}{303.133} & \multirow{4}{*}{311.133} & \multirow{4}{*}{321.553} & \multirow{4}{*}{311.554} \\
\hline & $\hat{\beta}=4.07103$ & & & & \\
\hline & $\hat{\sigma}=1.20307$ & & & & \\
\hline & $\hat{\theta}=2.86115$ & & & & \\
\hline \multirow{3}{*}{ LWD } & $\hat{\alpha}=0.413629$ & \multirow{3}{*}{344.475} & \multirow{3}{*}{350.475} & \multirow{3}{*}{358.29} & \multirow{3}{*}{350.725} \\
\hline & $\hat{\beta}=2.74561$ & & & & \\
\hline & $\hat{\theta}=1.025433$ & & & & \\
\hline \multirow{3}{*}{ HLGWD } & $\widehat{\omega}=1.842114$ & \multirow{3}{*}{342.308} & \multirow{3}{*}{348.308} & \multirow{3}{*}{353.519} & \multirow{3}{*}{348.432} \\
\hline & $\hat{\eta}=2.58473$ & & & & \\
\hline & $\hat{\gamma}=3.083311$ & & & & \\
\hline
\end{tabular}

Table 3. Estimated-2l, AIC, BIC, and CAIC for Dataset 2

\begin{tabular}{|c|c|c|c|c|c|}
\hline Distribution & Estimates & $-2 l$ & AIC & BIC & CAIC \\
\hline \multirow{4}{*}{ FWD } & $\hat{\alpha}=2.44162$ & \multirow{4}{*}{48.46} & \multirow{4}{*}{56.46} & \multirow{4}{*}{65.032} & \multirow{4}{*}{57.1496} \\
\hline & $\hat{\beta}=0.84899$ & & & & \\
\hline & $\hat{\lambda}=3.53867$ & & & & \\
\hline & $\hat{k}=3.07125$ & & & & \\
\hline \multirow{4}{*}{ GNEXWD } & $\hat{\alpha}=1.0843$ & \multirow{4}{*}{53.08} & \multirow{4}{*}{61.08} & \multirow{4}{*}{63.509} & \multirow{4}{*}{61.7696} \\
\hline & $\hat{\beta}=2.67261$ & & & & \\
\hline & $\hat{\sigma}=3.11944$ & & & & \\
\hline & $\hat{\theta}=1.02126$ & & & & \\
\hline \multirow{3}{*}{ LWD } & $\hat{\alpha}=0.908874$ & \multirow{3}{*}{62.63} & \multirow{3}{*}{68.63} & \multirow{3}{*}{77.202} & \multirow{3}{*}{69.0367} \\
\hline & $\hat{\beta}=2.75034$ & & & & \\
\hline & $\hat{\theta}=1.41351$ & & & & \\
\hline \multirow{3}{*}{ HLGWD } & $\widehat{\omega}=2.46512$ & \multirow{3}{*}{63.557} & \multirow{3}{*}{69.557} & \multirow{3}{*}{78.13} & \multirow{3}{*}{69.9837} \\
\hline & $\hat{\eta}=2.63314$ & & & & \\
\hline & $\hat{\gamma}=0.84273$ & & & & \\
\hline
\end{tabular}




\section{Conclusions}

A new generalized form of Weibull distribution known as FWD has been developed. Statistical properties such as moments, mode, quantile function and different types of entropy of the distribution are studied. The $p d f, c d f$ and limiting behavior of order statistics of FWD have been optimized. The applications of FWD to the two real datasets show the superiority of FWD over other related distributions. This study will provide a useful tool to analyze and modeling of datasets in Mechanical Engineering sciences and other related field.

\section{Acknowledgement}

The authors thank every person who helped them to complete this paper.

\section{REFERENCES}

[1] Ahmad, Z., Hamid, S.M. and Hussain, Z. Generalized new extended weibull distribution with real life application. MAYFEB Journal of Materials Science, 2017, 3(2): 117-128.

[2] Al-Aqtash, R., Lee, C. and Famoye, F. Gumbel-weibull distribution: Properties and applications. Journal of Modern Applied Statistical Methods, 2014, 13(2):11-23.

[3] Alzaatreh, A., Lee, C. and Famoye, F. A new method for generating families of continuous distributions. Metron, 2013, 71(1): 63-79.

[4] Anwar, M. and Bibi, A. The half-logistic generalized weibull distribution. Journal of Probability and Statistics, 2018, 21(4): 227-236.

[5] Block, H.W., Savits, T.H., Singh, H. The Reverse Hazard Rate Function. Probability in the Engineering and Informal Sciences, 1998, 12, 69-90.

[6] Cordeiro, G.M., Afify, A.Z., Yousof, H.M., Cakmakyapan, S. and Ozel, G. The Lindley weibull distribution: properties and applications. Anais da Academia Brasileira de Ciências, 2018, 90(3): 2579-2598.

[7] Cordeiro, G.M., Saboor, A., Khan, M.N., Provost, S.B. and Ortega. E.M.M. The transmuted generalized modified weibull distribution. Filomat, 2017, 31(5): 1395-1412.

[8] Galambos, J. The asymptotic theory of extreme order statistics. R.E. Krieger Pub. Co., 1987.

[9] Gumbel, E.J. Statistics of Extremes. Columbia University
Press, N.Y., 1958.

[10] Kapoor, K.C. and Lamberson, L.R. Reliability in Engineering Design. John Wiley \& Sons, 1977.

[11] Lai, C.D., Murthy, D.N. and Xie, M. Weibull distributions and their applications. Springer handbook of engineering statistics, 2006, 63-78.

[12] Mahmoud, M.R. and Mandouh, R.M. On the transmuted Fréchet distribution. Journal of Applied Sciences Research, 2013, 9(10):5553-5561.

[13] Mead, M.E., Afify, A.Z., Hamedani, G.G. and Ghosh, I. The beta exponential Fréchet distribution with applications. Austrian Journal of Statistics, 2017, 46(1): 41-63.

[14] Nadarajah, S. and Kotz, S. The exponentiated Fréchet distribution. Interstat Statjournals, 2003, 24(2): 31-42.

[15] Nelson H. and Wayne, L. How to Analyze Reliability Data, ASQ Basic Reference in Quality Control. Statistical Techniques, 1983, 6 .

[16] Nichols, M.D. and Padgett, W.J. A Bootstrap Control Chart for Weibull Percentiles. Quality and Reliability Engineering International, 2006, 22141-151, http://dx.doisorg/10.1002/q re.691.

[17] Pobocikova, I., Sedliackova, Z. and Michalková, M. Transmuted weibull distribution and its applications. In MATEC Web of Conferences, EDP Sciences, 2018, 157: 70-82.

[18] Sengupta, D., Singh, H., Nanda, A.K. The proportional Reversed Hazard Rate Model. Technical Report, Indian Statistical Institute, Calcutta, 1999.

[19] Sica, C. and Luko, S. Correction Factors for use with The Method of "Sudden Death" Weibull Analysis. SAETechnical Paper 982031, off Highway Conference, Sept. 1998.

[20] Silva, R.V. Da., Andrade, T.A.N. De., Maciel, D.B.M., Campos, R.P.S. and Cordeiro, G.M. A new lifetime model: The gamma extended Fréchet distribution. JSTA, 2013, 12(1): 39-54.

[21] Smith, R.L. and Naylor, J.C. A Comparison of Maximum Likelihood and Bayesian Estimators for the three parameters Weibull Distribution. Applied Statistics, 1987, 36, 358-369, http://dx.doi.org/10.2307/2347795.

[22] Tabass, M.S., Borzadaran, G.R.M. and Amini, M. Renyi entropy in continuous case is not the limit of discrete case. Mathematical Sciences and Applications e-Notes, 2016, 4(1): 113-117.

[23] Yousof, H.M., Afify, A.Z., Ebraheim, A.N., Hamedani, G.G. and Butt, N.S. On six-parameter Fréchet distribution: Properties and applications. Pakistan Journal of Statistics and Operation Research, 2016, 12(2): 281-299. 